\title{
Neurodegeneration, gliosis, and resolution of haemorrhage in neovascular age-related macular degeneration, a clinicopathologic correlation
}

\author{
Miaoling $\mathrm{Li}^{1,2} \cdot$ Rosa Dolz-Marco ${ }^{3,4,5} \cdot$ Jeffrey D. Messinger ${ }^{2} \cdot$ Daniela Ferrara $^{6} \cdot$ K. Bailey Freund ${ }^{3,4,7}$. \\ Christine A. Curcio $\mathbb{1}^{2}$
}

Received: 7 March 2020 / Revised: 7 April 2020 / Accepted: 14 April 2020 / Published online: 4 May 2020

(c) The Author(s), under exclusive licence to The Royal College of Ophthalmologists 2020

\begin{abstract}
Background To analyse cellular and spatiotemporal factors of neurodegeneration and gliosis in a patient with submacular haemorrhage $(\mathrm{SMH})$ secondary to type 1 macular neovascularization in neovascular age-related macular degeneration (nAMD).

Methods This is a case study and clinicopathologic correlation of an 84-year-old white man with nAMD treated with antiangiogenic drugs and photodynamic therapy during a 6-year follow-up. Eyes were recovered for histology $8.23 \mathrm{~h}$ after death. In vivo multimodal imaging including optical coherence tomography (OCT) and en face modalities was compared with ex vivo OCT and high-resolution histologic images, using a custom image registration procedure. SMH components were defined (intraretinal, subretinal, sub-retinal pigment epithelium (RPE), and dehemoglobinized blood). Neurodegenerative changes in each of these areas were described. One anonymous donor eye with haemorrhagic nAMD was also reviewed as a comparator.

Results By in vivo OCT, progressive resolution of the haemorrhage and gradual transformation of sub-RPE fluid to fibrous hyperreflective tissue, progressive macular atrophy, and variation in external limiting membrane (ELM) visibility were observed. Histology showed intense photoreceptor loss with preservation and self-adhesion of macular Müller glia resulting in ELM condensation. The comparator eye exhibited shed cone inner segments among subretinal erythrocytes.

Conclusion This is the most detailed clinicopathologic correlation of nAMD with SMH resolution to date, and the first in the OCT era. Our results reveal profound macular neurodegeneration and gliosis, signified by condensed ELM, soon after haemorrhage begins. Intensified OCT reflectivity of the ELM, an important retinal barrier, has potential as a biomarker for severe photoreceptor loss and gliosis.
\end{abstract}

These authors contributed equally: Miaoling Li, Rosa Dolz-Marco

Supplementary information The online version of this article (https:// doi.org/10.1038/s41433-020-0896-y) contains supplementary material, which is available to authorised users.

$\triangle$ Christine A. Curcio

christinecurcio@uabmc.edu

1 State Key Laboratory of Ophthalmology, Zhongshan Ophthalmic Center, Sun Yat-sen University, Guangzhou, China

2 Department of Ophthalmology and Visual Sciences, School of Medicine, University of Alabama at Birmingham, Birmingham, AL, USA

3 Vitreous Retina Macula Consultants of New York, New York, NY, USA

\section{Introduction}

In eyes with neovascular age-related macular degeneration (nAMD), submacular haemorrhage (SMH) can occur suddenly, often resulting in some degree of irreversible vision loss. Large SMHs are characteristic of a growth pattern of

4 LuEsther T Mertz Retinal Research Center, Manhattan Eye, Ear and Throat Hospital, New York, NY, USA

5 Unit of Macula, Oftalvist Clinic, Valencia, Spain

6 Genentech, South San Francisco, CA, USA

7 Department of Ophthalmology, New York University School of Medicine, New York, NY, USA 
sub-RPE neovascularization in which a branching vascular network feeds dilated vascular elements prone to exudation, a pattern recently termed 'aneurysmal type 1 macular neovascularization' (AT1) [1, 2]. When SMH occurs in nAMD, it may accumulate between the neurosensory retina and retinal pigment epithelium (RPE), or under the RPE. A small haemorrhage $(<1$ disk diameter (DD)) is considered part of the typical disease presentation, whereas larger haemorrhages are considered SMH. They are classified by size: small (1-4 DD); medium ( $\geq 4 \mathrm{DD}$, within the vascular arcades); and massive (extending beyond the arcades). SMHs are usually $<500 \mu \mathrm{m}$ thick and are considered thick if $>500 \mu \mathrm{m}$ [3]. We use this terminology, although this definition does not include intraretinal haemorrhage, as seen in the index case of this report.

The natural history of SMH is poor, including visual impairment associated with progression to fibrosis, RPE tears, or atrophic scars $[4,5]$. Haemorrhage thickness has been correlated with prognosis $[4,5]$, although not directly to the final visual acuity. SMH secondary to nAMD entails worse prognosis than haemorrhage associated with other conditions [5]. Factors underlying limited visual improvement include the presence of sub-RPE blood, a tendency for scarring, and other concomitant AMD-related retinal and RPE abnormalities. In experimental SMH, photoreceptors are damaged as soon as $1 \mathrm{~h} \mathrm{[6]} \mathrm{after} \mathrm{exposure} \mathrm{to} \mathrm{whole}$ blood, irreversibly within $24 \mathrm{~h} \mathrm{[7],} \mathrm{due} \mathrm{to} \mathrm{fibrin} \mathrm{contraction}$ and a diffusion barrier imposed by the clot.

Human macula exhibits distinctive forms of neurodegeneration and gliosis [8-10]. Unique to this region are photoreceptors with long axons interleaved with Müller glia outer trunks in the Henle fibre layer [11-13]. Histologically validated, longitudinal imaging anchored on optical coherence tomography (OCT) [14, 15] has recently confirmed early observations $[16,17]$ that the border of atrophy in the photoreceptor layers is a descent of the external limiting membrane (ELM) towards Bruch's membrane. The ELM represents junctional complexes between Müller cells and photoreceptors, a physiologic barrier to fluid flow, and a regulator of the photoreceptor environment [18]. When the ELM curves downward as in complete RPE and outer retinal atrophy (cRORA) [19], the normally vertical Müller glia re-orient horizontally and strongly express glial fibrillary acidic protein, a marker for reactive gliosis [20, 21]. Previously investigated as a predictor of visual acuity in macular atrophy [22-25], the ELM thus also has potential as a readout of photoreceptor survival after insult [14].

Histopathology of haemorrhage resolution in human retina can add new knowledge to that learned from experimental animals lacking maculae [6, 7], with the benefit of also informing the interpretation of clinical OCT. Herein we present histology of a clinically documented case of AT1 nAMD with a medium-sized, multi-layered SMH that was treated with non-surgical methods, thus allowing insight into photoreceptor destruction. We analyse cellular and spatiotemporal factors of neurodegeneration using a novel procedure to co-register multimodal images, correlating in vivo images through a 6-year follow-up and ex vivo high-resolution epoxy resin histology. Our data show remarkable photoreceptor loss with preservation of the ELM and Müller glia.

\section{Methods}

\section{Compliance}

This study complied with the Health Insurance Portability and Accountability Act of 1996 and adhered to the Tenets of the Declaration of Helsinki. Clinical review was approved by the Institutional Review Board of the Manhattan Eye, Ear and Throat Hospital/North Shore Long Island Jewish Hospital. The histopathology study was approved by the Institutional Review Board at the University of Alabama at Birmingham.

\section{In vivo multimodal imaging analysis}

An 84-year-old white man diagnosed with nAMD in his right eye and non-neovascular AMD in his left eye underwent comprehensive ophthalmologic examination and multimodal imaging. As separately detailed and summarised in the 'Results' [26], this patient received care at Vitreous Retina Macula Consultants of New York for 6 years. In 2008 and 2011 he underwent photodynamic therapy (PDT) in his right eye, in combination with intravitreal anti-vascular endothelial growth factor (VEGF) (55 injections over a 78-month follow-up). His last intravitreal treatment and clinical examination was in April 2015, 14 weeks before death. Clinically, the left eye demonstrated peripapillary subretinal drusenoid deposits and signs of diffuse outer retinal atrophy, but these features were not available for histology due to poor preservation.

Images available for review included colour fundus photography (CFP), red free (RF), fluorescein angiography (FA), indocyanine green angiography (ICGA), eye-tracked spectraldomain OCT, and near-infra-red reflectance (NIR). CFP and RF images were acquired using the Topcon TRC 501× fundus camera (Topcon Imagenet, Tokyo, Japan). FA and ICGA images were acquired using Topcon TRC 501x fundus camera or Heidelberg Spectralis HRA + OCT (Heidelberg Engineering, Heidelberg, Germany). OCT and NIR imaging were performed using the Spectralis. OCT imaging utilised eye tracking and image registration, with a customised evaluation field between $20^{\circ} \times 15^{\circ}$ and $30^{\circ} \times 25^{\circ}$ and with 
Automatic Real Time (ART) values between 10 and 30, and quality value between 17 and $38 \mathrm{~dB}$.

\section{Ex vivo multimodal imaging and histopathologic analysis}

The patient died due to subdural haematoma after a fall. Eyes were recovered $8.23 \mathrm{~h}$ after death by personnel of the Eye-Bank for Sight Restoration (New York, NY). Globes were opened anteriorly with encircling cuts at the limbus and immersed in $10 \%$ neutral buffered formalin, shipped on wet ice overnight, and transferred to $2 \%$ glutaraldehyde and $1 \%$ paraformaldehyde in $0.1 \mathrm{M}$ phosphate buffer. Ex vivo OCT and NIR $(787 \mathrm{~nm})$ reflectance imaging of the preserved globe were obtained prior to histologic processing, as described [27]. An 8-mm-diameter full-thickness tissue punch centred on the fovea was post-fixed with osmium tannic acid paraphenylenediamine to preserve extracellular lipids, embedded in epoxy resin, sectioned at $0.8 \mu \mathrm{m}$ thickness, and stained with toluidine blue [26].

To provide seamless multi-scale review without switching between microscope objectives, sections were scanned in their entirety using a $60 \times$ oil-immersion objective (1.4 numerical aperture), a robotic microscope stage, and slide scanning software (Olympus VSI 120, CellSens; Olympus, Center Valley PA). For detailed evaluation, scanned images were viewed on a monitor at magnifications up to $1240 \mathrm{X}$ using ImageJ (https://imagej.nih.gov/ij/download.html). Photomicrographs were composited for figures with adjustments for exposure, contrast, and background colour (Photoshop CS6, Adobe Systems, USA).

\section{Integration of in vivo imaging, over time, with histology}

Our goal was to correlate histology with specific macular regions on in vivo imaging, each with distinct features of neovascularization, exudation, and haemorrhage over the course of follow-up. This goal was implemented by locating these areas on in vivo en face images acquired at different time points from different devices, projecting them onto in vivo OCT B-scans, and then from there to ex vivo tissues sections, as described [26], using two custom ImageJ plugins (Register Barycentric, EnFace2OCT_Markup).

In brief, all in vivo en face images were aligned to a reference NIR using barycentric alignment [28]. Retinal vessels were used to align en face images of all imaging modalities, two at a time to the NIR locator image used for OCT. Then, haemorrhagic areas on the aligned en face images were outlined manually in different colours [26] and simultaneously indicated in the B-scans by identically colour-coded vertical lines. Working in the other direction, histology was compared with ex vivo B-scans then to in vivo B-scans from the last clinic visit with OCT (December 2014, 8 months before death) by comparing shapes of entire sections and scans, as described above. Atrophic retina remained attached to the underlying support tissues in one area. We reduced the impact on alignment of torsion around this attachment by considering the spatial ordering of tissue features as (superior to inferior and nasal to temporal), across scans and across sections. Finally, we accounted for shrinkage of detached retina.

\section{Comparator donor eye}

Histopathologic features of neurosensory retina in the index eye were compared with an eye with nAMD from the Project MACULA website of AMD histopathology with a haemorrhage that was smaller than SMH as defined [3]. This eye had undergone ex vivo colour fundus imaging and OCT as described [29]. The comparator case was accessioned in 2001, i.e. before the anti-VEGF era. Presence of basal linear and laminar deposits supported the histologic diagnosis of AMD. Subretinal drusenoid deposits were not detected.

\section{Results}

Figure 1 shows different components of the AT1 lesion at baseline on in vivo multimodal imaging, including CFP (Fig. 1a), RF (Fig. 1b), ICGA (Fig. 1c, mid phase) and FA (Fig. 1d, late phase). Clinical features included intraretinal, subretinal, and sub-RPE haemorrhage/fluid, dehemoglobinized haemorrhage, and as detailed separately [26], aneurysms/polyps and a peripapillary branching vascular network. At baseline, a haemorrhage area greater than 4 DD was confined to the macula without extending to the temporal vascular arcades, meeting the criterion for mediumsized SMH [3]. Subfoveal haemorrhage thickness was 201 $\mu \mathrm{m}$, and dehemoglobinized blood was the thickest component $(400 \mu \mathrm{m})$. Intraretinal haemorrhage appeared red and radiated outward from the foveal centre in a pattern consistent with localisation to the HFL. This haemorrhage extended mostly into the outer nuclear and outer plexiform layers with some hyperreflective material also within the inner nuclear layer. Subretinal haemorrhage was bright red and diffusely distributed. Sub-RPE haemorrhage heterogeneously brownish-red, diffusely distributed, and mixed with serous fluid. Dehemoglobinized blood was yellowishwhite with sharply delimited borders.

During the follow-up, resolution of subretinal and intraretinal haemorrhage and progressive transformation of the sub-RPE fluid to hyperreflective fibrous tissue (Fig. 2) were observed by OCT. At baseline, areas of subretinal haemorrhage (Fig. 2a) and fluid, intraretinal haemorrhage 
Fig. 1 Lesion components at baseline (October 2008), in aligned en face images. Lesion boundaries are not shown for illustrative clarity. Components are numbered and color-coded for correspondence with Fig. 2. 1, sub-retinal pigment epithelium (RPE) fluid; 2 , subretinal dehemoglobinized hemorrhage; 3 , hemorrhage in Henle fiber layer; 4, sub-RPE hemorrhage and fluid; 5 , subretinal hemorrhage; 6 , subRPE hemorrhage; 7, aneurysm/ polyp; 8,9 , peripapillary neovascularization. a Color fundus photograph; $\mathbf{b}$ red-free image; c mid phase indocyanine green angiogram; $\mathbf{d}$ late phase fundus fluorescein angiogram. Aneurysmal type 1 lesions 8-9 were best seen in a separately published late phase indocyanine green angiogram [26]; these are included here for completenes.

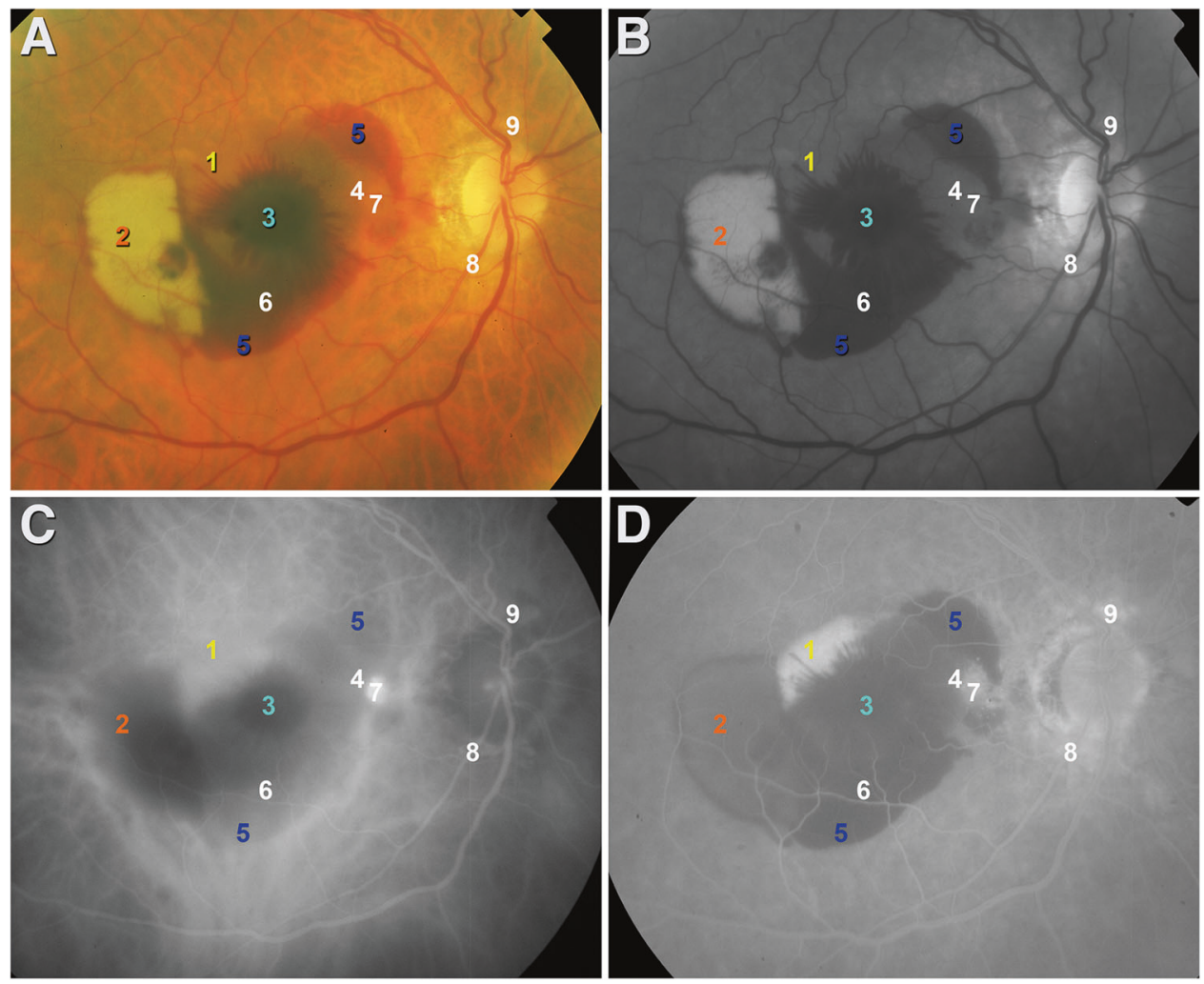

(Fig. 2b) showed an intensified (more reflective) ELM that progressed to outer retinal atrophy (ORA) and undetectable ELM after resolution (Fig. 2c, d). At baseline, dehemoglobinized haemorrhage appeared as a homogeneously hyperreflective subretinal material, and the ELM was not visible (Fig. 2b, orange arrowhead). As this subretinal material was resorbed, progressive outer retinal degeneration was evident. Over the follow-up, areas of RORA with choroidal hypertransmission on OCT B-scans were observed in areas of subretinal and Henle fibre layer haemorrhage (Fig. 2c, d). The ELM variably presented as continuous and homogeneously hyperreflective (normal, A1, B1), thick hyperreflective (intensified, A2, B2), or not visible due to a diffuse and heterogeneous reflectivity extending from the RPE band through the ONL (A3, B3).

Outer retina was studied at cellular and subcellular levels with high-resolution histology (Figs. 3, 4, S1, S2). ELM status varied remarkably with photoreceptor content (Fig. 3). In areas where numerous photoreceptor inner segments passed through the ELM, the ELM exhibited its normal light microscopic appearance of a discontinuous thin line (Fig. 3a). In areas where IS and OS were absent due to total or near-total photoreceptor depletion, fine processes of Müller cells made junctions among themselves, and the ELM condensed into a nearly continuous line at this magnification (Fig. 3c). An intermediate stage of ELM condensation was also defined (Fig. 3b). This prominent histologic variation could not be definitively correlated to variation in OCT appearance at the time points examined (2009 OCT, 2014 OCT, 2015 histology).

In areas with intraretinal haemorrhage and serohaemorrhagic PED, depletion of photoreceptors from the ONL left a thickened HFL consisting of only Müller cell processes (Figs. 2b, 3c, 4d). HFL/ONL dyslamination, i.e. loss of discrete layers due to inward translocation of photoreceptor cell bodies [12], was also seen. In areas with subretinal and dehemoglobinized haemorrhage (Fig. 4a-c, e, f) were also other cells (phagocytes, re-positioned Müller cells, migrated RPE), and debris. Other findings included a hyperreflective foci within ORT that correlated to an entubulated RPE cell (Fig. S1, yellow arrowhead). Areas of ordered Müller cell fibres and atrophic ONL lacking cellular infiltration, with an ELM descent on each side (Fig. S2, green arrowheads), correlated to hyporeflective wedges.

Retinal findings in the index eye, seen histologically 4 years after the last clinically documented haemorrhage, were compared with a donor eye with nAMD and subretinal haemorrhage (Fig. 5). Because blood was apparent in ex vivo CFP of the comparator eye (Fig. 5a) and erythrocytes were present in the subretinal space (Fig. 5c), we concluded that this eye represented an earlier time point in the resolution of haemorrhage than that of the index eye. Nevertheless, there was noticeable loss of photoreceptor inner and outer segments, inner segments containing 
Fig. 2 Resolution of subretinal and intraretinal hemorrhage and exudation; variability of the external limiting membrane (ELM). The ELM was seen as normal, condensed, and not visible on crosssectional appearance on optical coherence tomography scans (a-b). a The subretinal exudation and trace amount of hemorrhage remained (blue frame) 3 months after the first photodynamic therapy (2009). The reflection of the associated ELM was intensified (magnified in A2, blue arrowhead) compared with the one visible in the temporal side (green frame, magnified in $\mathrm{A} 1$, green arrowhead). b Subretinal hyperreflectivity corresponding to the area of dehemoglobinized hemorrhage (orange arrowhead), and the hemorrhage in the HFL accompanied with intensification of ELM (teal frame, magnified in B2), were present. Areas with undetectable ELM (yellow frames, magnified in A3, B3) exhibited diffusely homogeneous hyperreflectivity that subsumed bands from RPE to HFL. c The follow-up scan of (a). The exudation and hemorrhage resolved, and the ELM was absent at the visit of 5 years later (2014). d The followup scan of (b). The hemorrhage resolved, and the ELM was absent.
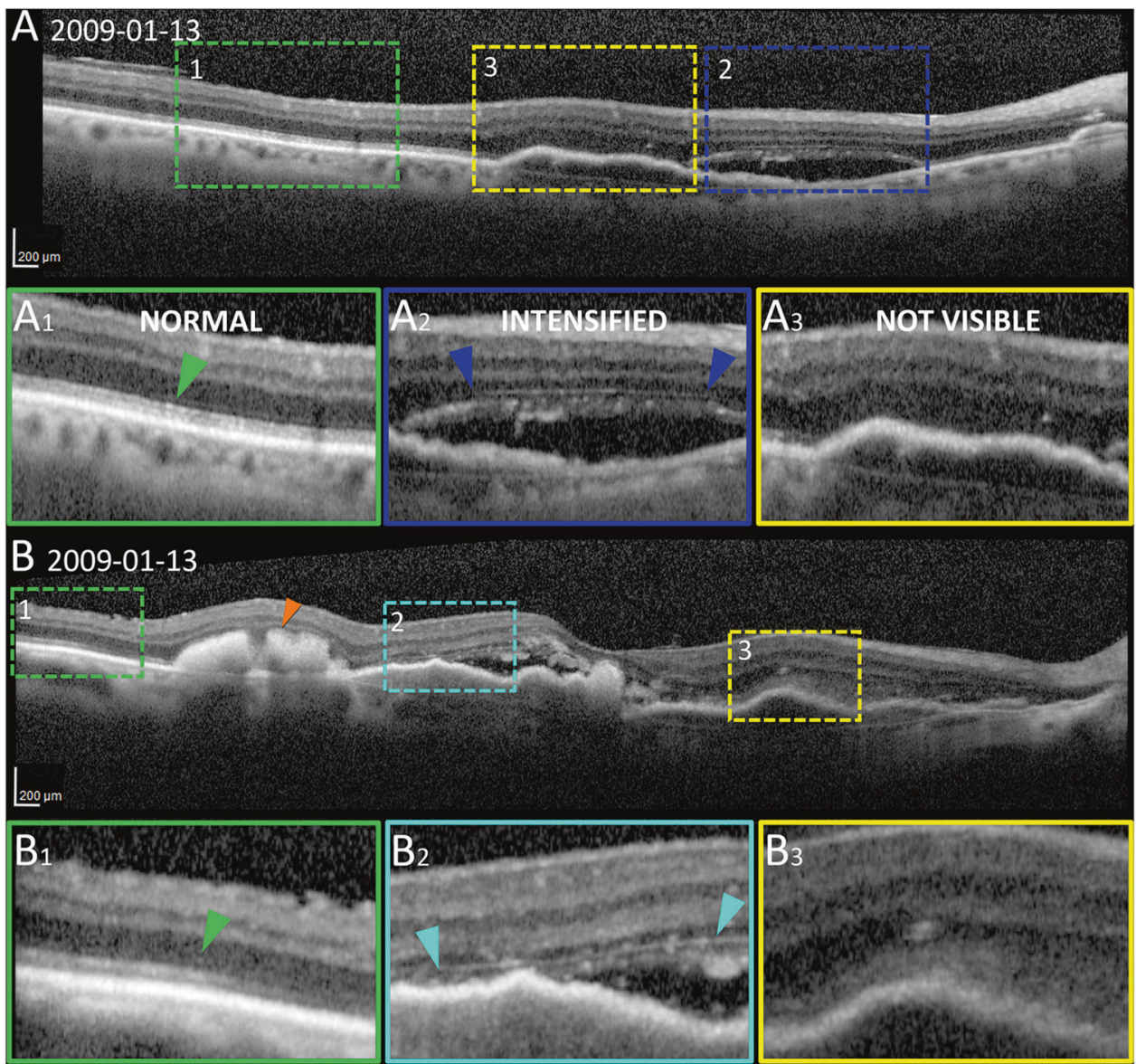

C 2014-06-25

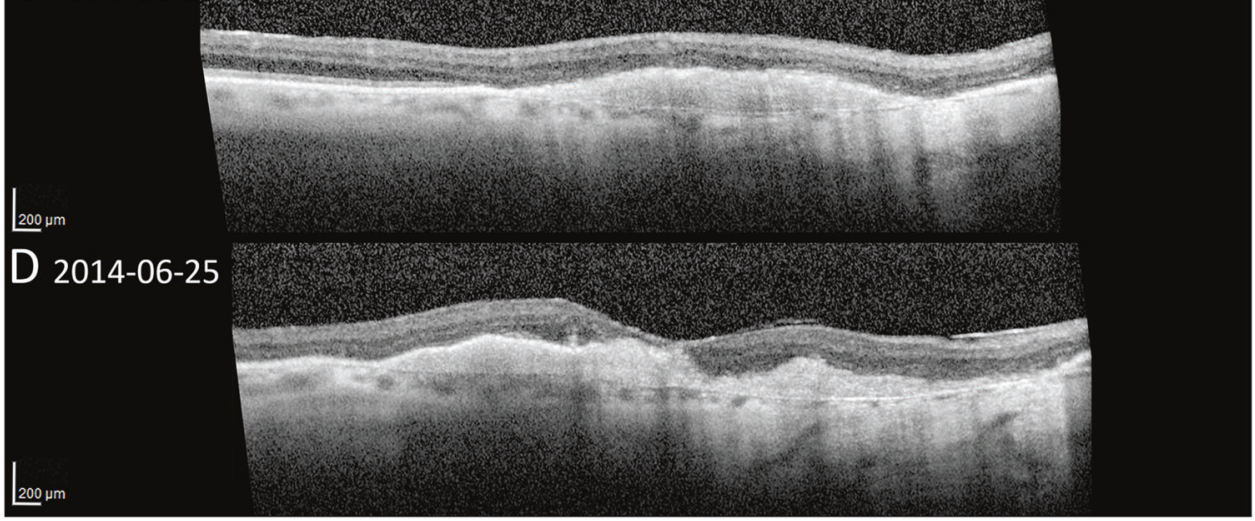

scattered and fragmented mitochondria shed into the subretinal space (Fig. 5e) [9], dyslamination of the HFL/ONL (Fig. 5e), Müller cell bodies repositioning within a distorted INL (not shown), and ORT in atrophic retina overlying the fibrovascular scar (not shown). Similar to this comparator eye, the index eye exhibited absent photoreceptors or scattered cone nuclei in the area of RPE atrophy, persistence of Müller cell processes after photoreceptor depletion, dyslaminate HFL/ONL, and ELM condensation. Unlike this comparator eye, the index eye exhibited depletion of photoreceptor nuclei with persistence of Müller cells in HFL in an area of still-present RPE (Fig. 4d), ORTs in both nonatrophic (Fig. 4c, green arrowhead) and atrophic areas, abundant foreign cells/cellular debris in the HFL/ONL, and no evidence of shed inner segments.

\section{Discussion}

We present a detailed correlation of SMH in nAMD, the first in the OCT era. In the index case, we found photoreceptor damage not only in areas meeting criteria for 


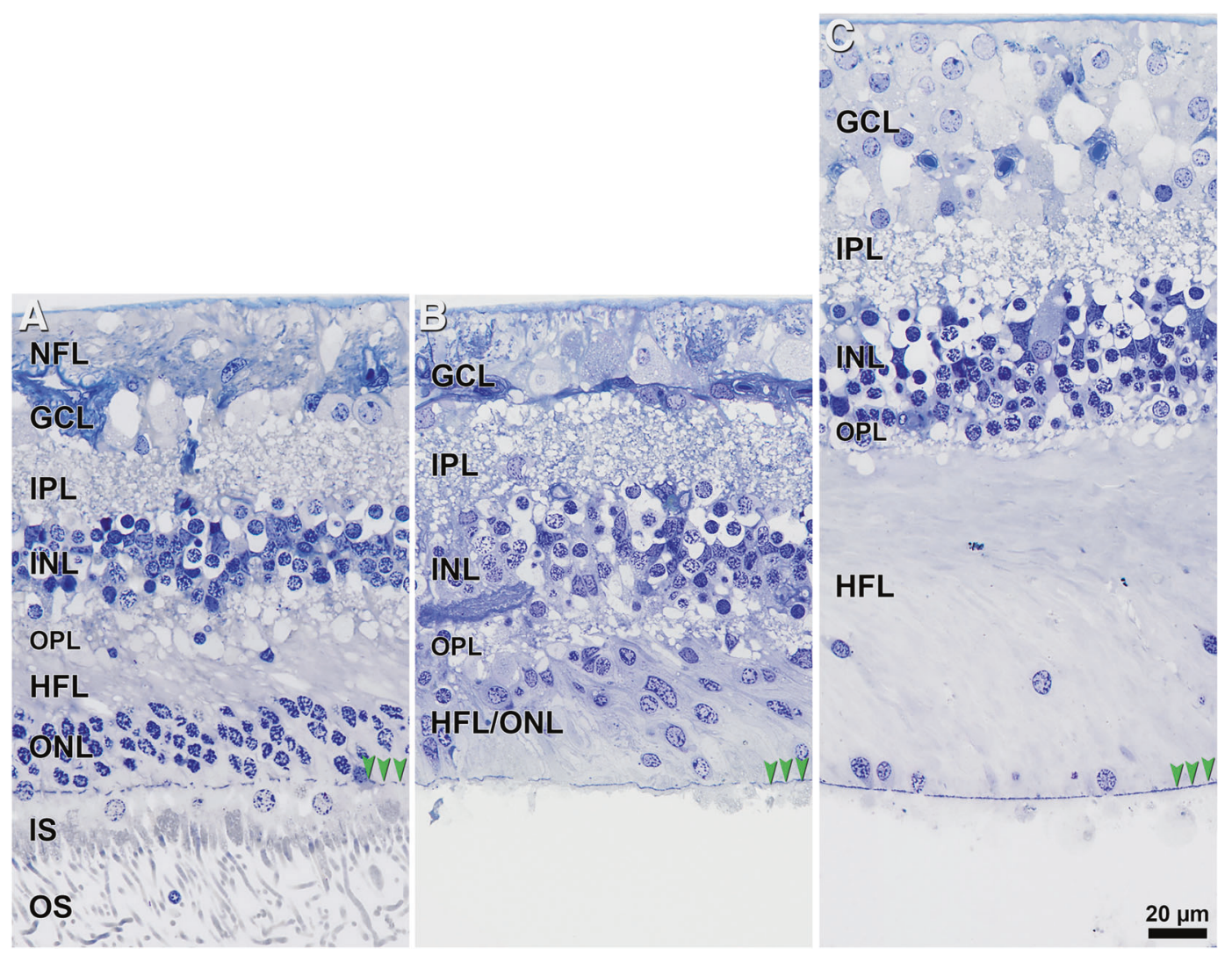

Fig. 3 Histologic condensation of the external limiting membrane (ELM). NFL nerve fiber layer, GCL ganglion cell layer, IPL inner plexiform layer, INL inner nuclear layer, OPL outer plexiform layer, HFL Henle fiber layer, ONL outer nuclear layer, IS inner segment, OS outer segment. External limiting membrane, green arrowheads. Scale bar in (c) applies to all panels. All panels are from the studied eye. a ONL is thick with some ectopic rod photoreceptor nuclei in the HFL and cone nuclei in the inner segments. The ELM is perforated by intact
IS and appears normal. b With IS largely absent, the ELM is mostly composed of fine processes of Müller glia contacting each other and thus appears condensed. The HFL/ONL is dyslaminate. c Photoreceptor nuclei are mostly depleted, so ONL is absent and only a thick layer of Müller glia outer trunks remain. The ELM appears highly condensed. Distance of the scaled areas from the center of section: A$1756 \mu \mathrm{m}, \mathrm{B}-2283 \mu \mathrm{m}$, and C-606 $\mu \mathrm{m}$.
cRORA, where only scattered cone cell bodies remained, but also over non-atrophic RPE (ORA), where the HFL consisted almost exclusively of Müller glia (Fig. 4d). We suggest that severe neurodegeneration and gliosis in nonatrophic areas were due to exudation and haemorrhage rather than RPE failure. In our study, we infer that Müller cell fibres persisting in the HFL are gliotic, extrapolating from the previously reported, marked up-regulation of glial fibrillary acidic protein in atrophic areas of eyes with advanced AMD [20, 21, 30]. An inherent limitation to histology is the snapshot view, often after a disease process has terminated. By adding a comparator case of a donor eye, we could see that inner segment shedding and HFL/ONL dyslamination is underway when blood is still present in the subretinal space (Fig. 5d, e).

High-resolution histology of the neurodegeneration of nAMD adds new detail to this literature. In analysing 176 human eyes by paraffin histology, Green and Key reported serosanguinous retinal detachments associated with loss of photoreceptors, ONL thinning, and INL sparing [31]. Studies of intact donor eyes noted concomitant absence of photoreceptors and RPE [32-34], and at the border of atrophy, fewer photoreceptor nuclei and shorter outer segments [33]. Excised submacular neovascular membranes had photoreceptors attached [35, 36] in $24 \%$ of specimens [37]. One of us (CAC) showed survival of cones over rods, both overlying fibrovascular scars subsequent to macular neovascularization (MNV) and in the intact photoreceptor layer peripheral to an ELM descent [38]. Recently we demonstrated shedding of cone inner segments into the subretinal space, distinguishable from subretinal drusenoid deposit in the same histologic sections, and containing degenerate mitochondria verified with electron microscopy [8].

Mechanistic knowledge about neurodegeneration following subretinal haemorrhage was attained experimentally through surgical introduction of autologous fresh whole blood into laboratory animals $[6,7,39]$. Notable findings 


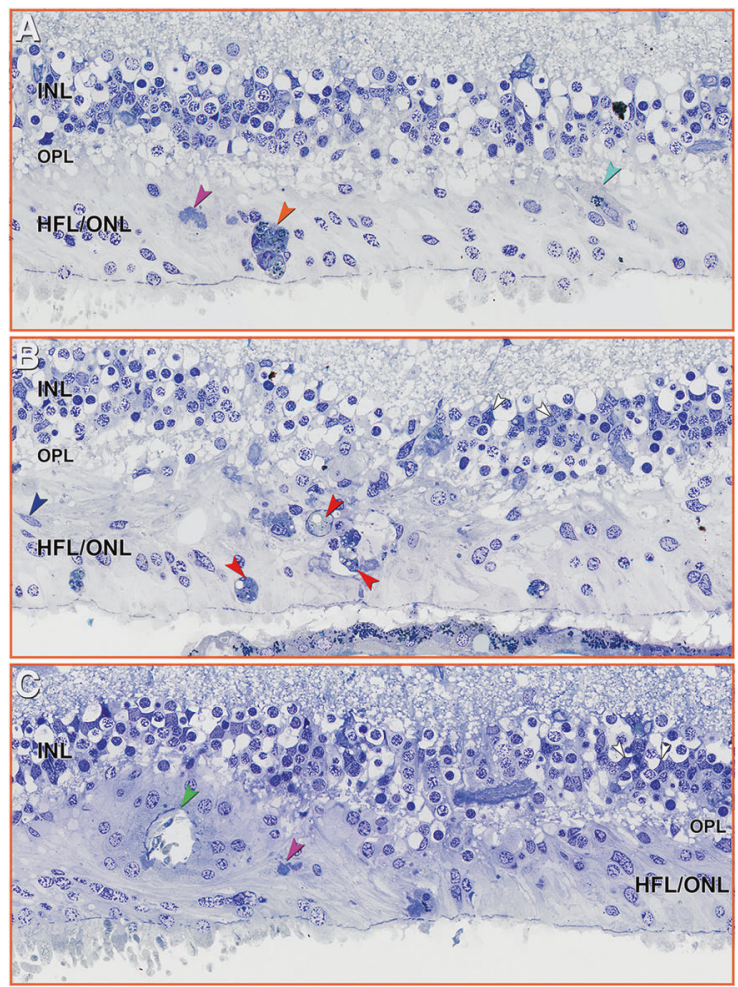

Fig. 4 Cells in Henle fiber layer (HFL)/outer nuclear layer (ONL) in areas with subretinal and intraretinal hemorrhage. IPL inner plexiform layer; INL inner nuclear layer; OPL outer plexiform layer. Scale bar in (f) applies to all panels. All panels are color-coded in accordance with Fig. 1: orange frame $(\mathbf{a}-\mathbf{c})$ areas with dehemoglobinized subretinal hemorrhage; teal frame (d) areas with intraretinal hemorrhage; blue frame (e, f)- areas with non-dehemoglobinized subretinal hemorrhage. In areas with subretinal hemorrhage $(\mathbf{a}-\mathbf{c}, \mathbf{e}, \mathbf{f})$, the cellular changes are more common than in areas with intraretinal

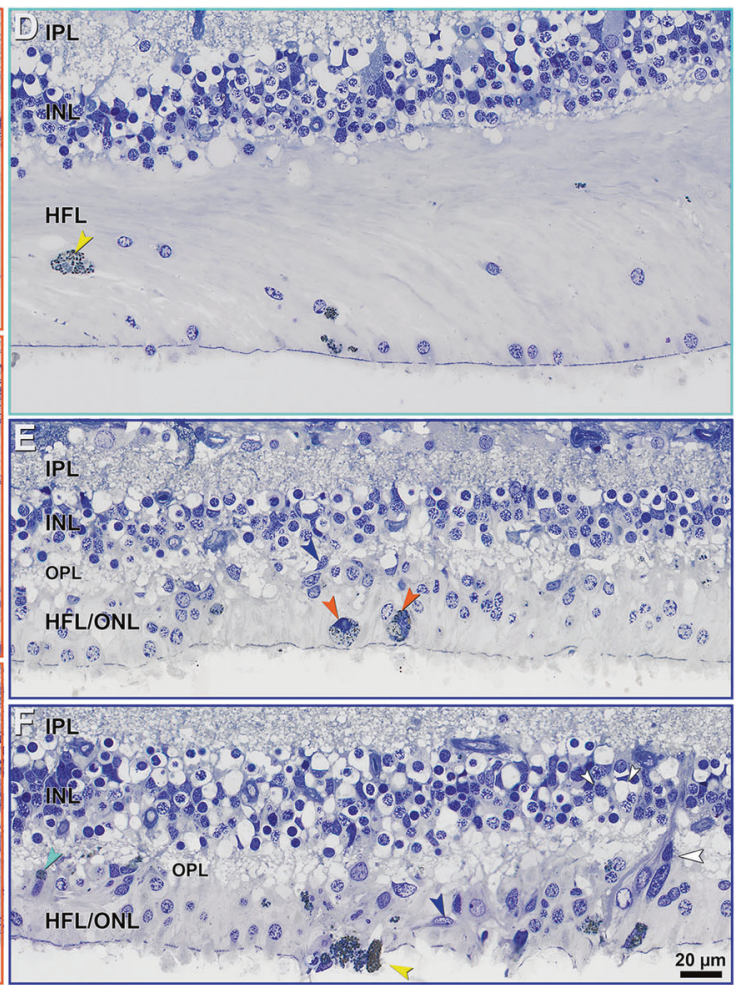

hemorrhage (d). In areas with intraretinal hemorrhage (d), the photoreceptor nuclei are mostly depleted, and the HFL is gliotic. Pink arrowheads, purple-stained debris; orange arrowheads, phagocytes with pigment granules; teal arrowheads, possible Müller cells with pigment granules; blue arrowheads, possible microglia; red arrowheads, phagocytes with lipids; green arrowhead, outer retinal tubulation; yellow arrowheads, migrated RPE cells; small white arrowheads, Müller cell bodies in INL; big white arrowheads, possible Müller cell bodies presenting in HFL/ONL with distortion of INL. included irreversible photoreceptor damage within $24 \mathrm{~h}$, due to mechanical disruption by the introduced blood and contraction of fibrin strands at $<1 \mathrm{~h}[6,7]$. Histochemically detectable iron attributed to engulfed erythrocytes appeared in the retina at 7 days, supporting a potential role for iron toxicity in the long term [7]. Lewis et al. [39] reported that early photoreceptor damage was confined to the haemorrhage area and included loss of ONL cells overlying the thickest blood, underscoring the importance of a barrier to diffusion from the choriocapillaris [7]. These latter authors also described lack of gliosis, preservation of INL, presence of intraretinal vacuolisation and cystoid spaces, and relative sparing of the RPE monolayer, with loss of apical specialisations that normally contact outer segments [6]. Lesser glial involvement in rabbits compared with human tissues could be due to species' differences in cellular response, shorter time points examined, or both. Previous investigators noted species' differences in vascular systems [40] and the absence of a macula, but did not comment what the latter meant for cellular composition and activities, e.g., Müller glia and photoreceptors with unique properties.

With OCT imaging precise localisation of subretinal and/ or sub-RPE components of SMH is possible, although posterior shadowing may limit the visualisation of deeper structures [3]. Our case demonstrates radiating intraretinal haemorrhage (Fig. 1a) in a case of AT1 diagnosed by ICGA (Fig. 1c). In typical cases with similar intraretinal haemorrhages, OCT shows cysts with diffusely hyperreflective contents in the inner aspect of the ONL [41]. This characteristic distribution suggests that the intraretinal blood first occupies the inter-axon space at the HFL, up to a limit. If the haemorrhage enlarges within the HFL, it can grow only in the centrifugal direction, creating the distinct, asterlike appearance [41]. In the presence of a radiating haemorrhage, which reportedly occurs in up to $14.2 \%$ of nAMD cases, mainly associated with AT1, it is recommended to check for AT1. A radial pattern within the HFL due to spontaneous haemorrhage of deep capillary plexus was recently described in macular telangiectasia type 2 [42]. 

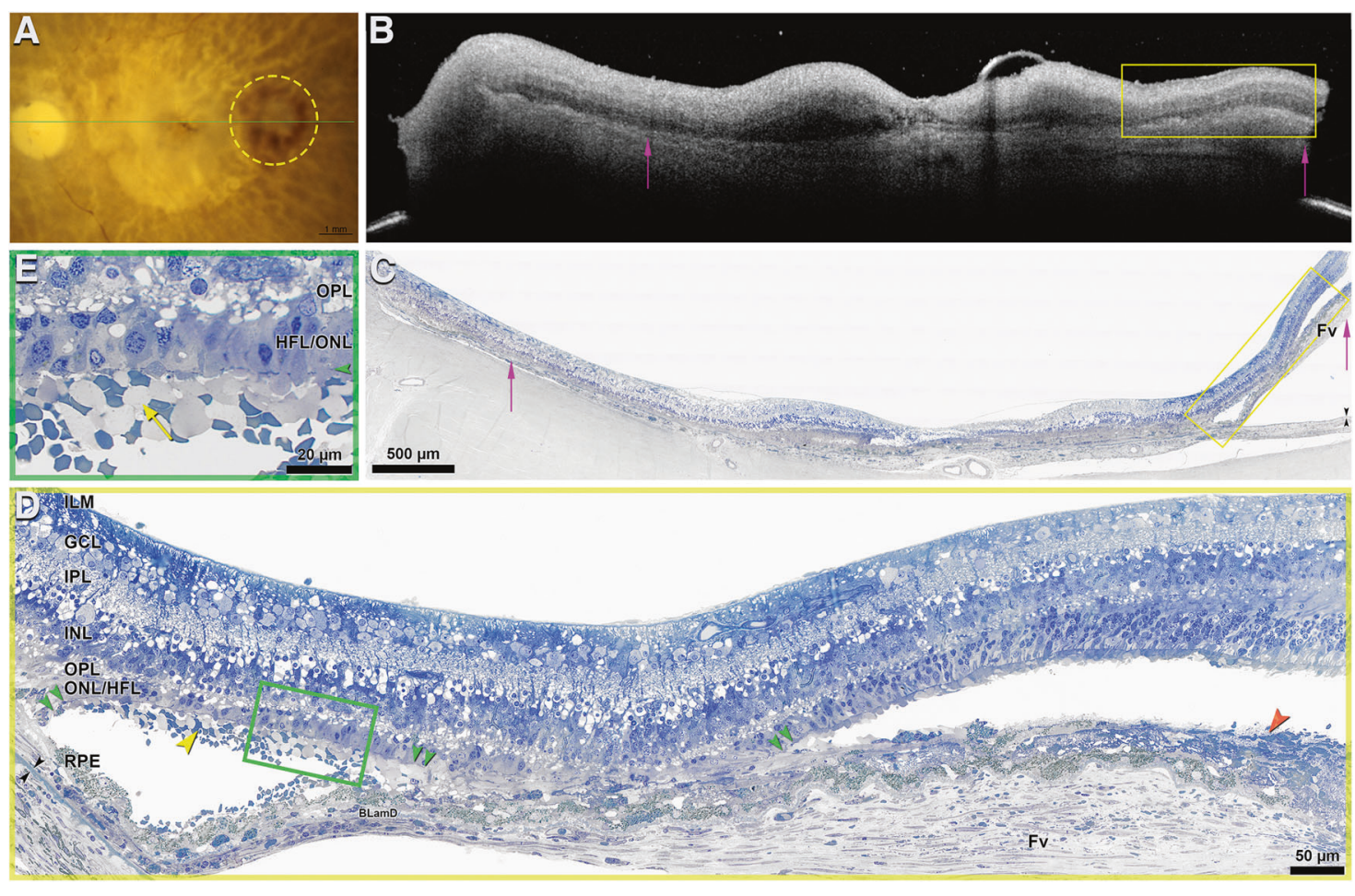

Fig. 5 Subretinal hemorrhage in the macula of a neovascular AMD eye. The scale bar in (c) applies to both (b) and (c). a. Ex vivo color fundus photograph shows macular atrophy and annular subretinal hemorrhage (yellow circle). Green line, scan level of the ex vivo optical coherence tomography (OCT). b Subretinal hemorrhage is hyperreflective (yellow frame) on ex vivo OCT. Hyperreflective type 1 macular neovascularization (MNV) is seen; the extent is indicated by pink arrows. c Yellow frame indicates area of subretinal hemorrhage. Pink arrows, extent of the fibrovascular scar (Fv); black arrowheads, Bruch membrane. The Fv correlates to the type $1 \mathrm{MNV}$ in (B). At this location, the retina is artifactually detached from the RPE, and the choroid, from the sclera. $\mathbf{d}$ Green frame, area detailed in panel e Orange arrowhead, subretinal hematoma mixed with fibrosis. At this location RPE is dysmorphic and continuous over a thin continuous BLamD, which overlies a fibrocellular scar with scattered blood cells. The overlying retina has dyslaminate HFL/ONL, and shortened inner segments, and absent outer segments. e Blood in the subretinal space is mixed with shed inner segments (yellow arrow), which harbor fragmented mitochondria [9]. Few inner segments extend beyond the external limiting membrane (green arrowhead). The HFL/ONL is dyslaminate. INL inner nuclear layer, OPL outer plexiform layer, HFL Henle fiber layer, ONL outer nuclear layer, RPE retinal pigment epithelium, BLamD basal lamina deposit.
SMH in nAMD has a poor visual prognosis, and different biomarkers influence the final visual outcome [43]. Ueda-Arakawa et al. found that despite frequent disruption of photoreceptor layers, 21 of 31 eyes demonstrated ELM preservation [44]. Our index case presented preserved ELM, due to Müller glia forming junctions among themselves in the setting of severe photoreceptor depletion. These results thus corroborate our similar findings in geographic atrophy secondary to AMD [12]. Although ELM integrity on OCT is considered a prognostic feature in retinal disease [45-47] including nAMD [22-25, 48], our current and published histology collectively underscore that ELM presence is not necessarily a sign of intact outer retina in the absence of independent evidence for photoreceptor survival.

These findings on ELM condensation have implications for the readout of clinical trials. For example, an ELM band visible over RPE cells transplanted into an atrophic area was considered evidence for possible integration with overlying retina [49]. In addition, inner segments are shed at an early stage of haemorrhage (Fig. 5), contributing to a disrupted ellipsoid zone (EZ) integrity on OCT. Intact EZ at baseline is indicative of visual prognosis in subretinal haemorrhage caused by nAMD [44] and high myopia (without choroidal degeneration) [50]. Taking together, our study supports EZ as a preferable biomarker for functioning photoreceptors over the ELM.

In the index eye, ELM intensification appeared during the first months of follow-up, then became invisible within areas of ORA and cRORA as exudation resolved. Yet a condensed ELM was apparent on histology. For this discrepancy between in vivo OCT and histology, we discuss three potential factors, in order: time elapsed between clinical presentation and histology analysis, clinical image quality including OCT axial resolution, and alignment of in vivo OCT with histology. During the 6 years' follow-up, only during the first months and in the presence of subretinal blood and fluid, ELM intensification was visible, 
suggesting that subretinal exudation or hyperreflective material reduced ELM visibility, rather than modifying ELM structure itself. ART values of OCT volumes in the index eye were $10-30$, and quality values were $17-38 \mathrm{~dB}$, considered adequate for ELM visibility [51], yet ELM visualisation on OCT was limited in areas of ORA, suggesting that better axial resolution may be required. Finally, it is possible that misalignment between in vivo OCT and histologic images may have introduced imprecision into this comparison, despite our precautions.

With the high-resolution histologic analysis, cellular changes in areas with subretinal haemorrhage (Fig. $4 \mathrm{a}-\mathrm{c}$, e, f) could be detected, including phagocytes (microglia/ macrophages), re-positioned Müller cells, migrated RPE cells and debris. Interestingly, intraretinal hyperreflective dots were identified in diseases with subretinal haemorrhage $[44,52]$ on OCT and presumed to be inflammation, macrophages, glia, or fibrin without direct evidence. Our study may provide clues to the cellular basis of the hyperreflective dots.

Our data can assist the development of new treatments for SMH by elucidating the influence of different haemorrhage components. SMH secondary to nAMD has been treated with tissue plasminogen activator (TPA), anti-VEGF drugs, intravitreal gas, and vitrectomy (with removal of neovascular membranes, macular translocation, RPE patch grafting, or SMH drainage) [3], all with the main goal of displacing blood away from the fovea. Management of acute massive SMH with intravitreal TPA, gas, and anti-VEGF therapy ('intravitreal triple therapy') may be an appropriate first-line treatment [3]. Visual improvement is limited, compared with eyes with absence of blood and presence of subretinal fluid, and varies with nAMD subtype, i.e. worst in type 1 aneurysmal, and best in type 3 [53]. Our histologic evidence for photoreceptor damage supports early intervention for massive SMH.

Of interest the patient received full-fluence PDT at initial presentation and once more while also receiving anti-VEGF medication [26]. The type $1 \mathrm{MNV}$ complex was very fibrous in this case [26]. It is uncertain how PDT specifically impacted findings in neurosensory retina, in part because four years passed between the second treatment and death [26]. Previous research has shown that photoreceptors are preserved after PDT using the standard parameters for treating AMD [54]. In experimental studies in cynomolgus monkeys, increased treatment intensity can cause damage to retina $[55,56]$. Type $1 \mathrm{MNV}$ is thought to compensate for outer retinal hypoxia and can sustain overlying RPE and photoreceptors, even during antiVEGF treatment [57, 58].

Strengths of this study include high quality clinical multimodal imaging with serial eye-tracked OCT, long-term follow-up, comprehensive histology, multi-scale viewing of OCT scans and histology, and current nomenclature for OCT and AMD pathology. Limitations include a 3-month gap between presentation and the first available spectral domain OCT, a 4-year gap between the last clinically documented haemorrhage and histopathology, and the overall complexity of haemorrhages in three tissue compartments. Nevertheless, this is the first detailed examination of human neurosensory retina following haemorrhage secondary to nAMD. Our data have implications for OCT interpretation. First, survival of only Müller cells in HFL/ ONL complicates interpretation of layer thickness measurements. Second, therapies and interventions should take into account a massive glial response, which may be underestimated with diagnostic technologies like CFP and dye angiography. Third, condensation of the ELM, a structure important in delimiting subretinal fluid and maintaining retinal integrity, can be investigated as an OCT biomarker for severe photoreceptor loss and gliosis in patients in whom neovascular lesions can be observed from when they start.

\section{Summary}

\section{What was known before}

- Existing histopathology of intact eyes with neurodegeneration in neovascular AMD is limited and does not show the detail needed for interpretation of multimodal OCT-based imaging.

- Our recent publications have underscored that neurodegeneration in the macula, with large cone photoreceptors and long Henle fibres, is distinct features relative to that in laboratory animals lacking maculae.

\section{What this study adds}

- Here we show with multi-modal clinical imaging haemorrhage in intraretinal, subretinal, and sub-RPE compartments and their long-term consequences.

- Via high-resolution epoxy-resin histology, retina associated with intraretinal haemorrhage had the most severe photoreceptor loss of the involved layers. In areas with complete photoreceptor depletion the external limiting membrane concomitantly condensed, as surviving Müller glia knitted together.

- Our data advance the interpretation of OCT, which is widely used for monitoring neovascular AMD, by demonstrating that presence of an ELM should not be construed as presence of photoreceptors without additional evidence. 
Funding This work was supported by Genentech/Hoffman-LaRoche. The funding organisation had no role in the design and conduct of this study. Purchase of the slide scanner was made possible by the Carl G. and Pauline Buck Trust.

\section{Compliance with ethical standards}

Conflict of interest No conflicting relationship exists for ML, JDM. RD-M is consultant to Heidelberg Engineering, Novartis and Allergan and receives research support form Genentech, Heidelberg Engineering, Novartis, and Roche. KBF is a consultant for Optovue, Heidelberg Engineering, Zeiss, Genentech, Novartis, and Allergan. He receives research support from Genentech/Roche. DF is an employee of Genentech and has stock/stock options of Roche. CAC receives research support from Heidelberg Engineering and owns stock of MacRegen.

Publisher's note Springer Nature remains neutral with regard to jurisdictional claims in published maps and institutional affiliations.

\section{References}

1. Yannuzzi LA, Sorenson J, Spaide RF, Lipson B. Idiopathic polypoidal choroidal vasculopathy (IPCV). 1990. Retina. 2012;32 Suppl 1:1-8.

2. Dansingani KK, Gal-Or O, Sadda SR, Yannuzzi LA, Freund KB. Understanding aneurysmal type 1 neovascularization (polypoidal choroidal vasculopathy): a lesson in the taxonomy of "expanded spectra' - a review. Clin Exp Ophthalmol. 2018;46:189-200.

3. Stanescu-Segall D, Balta F, Jackson TL. Submacular hemorrhage in neovascular age-related macular degeneration: a synthesis of the literature. Surv Ophthalmol. 2016;61:18-32.

4. Scupola A, Coscas G, Soubrane G, Balestrazzi E. Natural history of macular subretinal hemorrhage in age-related macular degeneration. Ophthalmologica. 1999;213:97-102.

5. Bennett SR, Folk JC, Blodi CF, Klugman M. Factors prognostic of visual outcome in patients with subretinal hemorrhage. Am J Ophthalmol. 1990;109:33-7.

6. Toth CA, Morse LS, Hjelmeland LM, Landers MB. Fibrin directs early retinal damage after experimental subretinal hemorrhage. Arch Ophthalmol. 1991;109:723-9.

7. Glatt H, Machemer R. Experimental subretinal hemorrhage in rabbits. Am J Ophthalmol. 1982;94:762-73.

8. Schaal KB, Freund KB, Litts KM, Zhang Y, Messinger JD, Curcio CA. Outer retinal tubulation in advanced age-related macular degeneration: Optical coherence tomographic findings correspond to histology. Retina. 2015;35:1339-50.

9. Litts KM, Messinger JD, Freund KB, Zhang Y, Curcio CA. Inner segment remodeling and mitochondrial translocation in cone photoreceptors in age-related macular degeneration with outer retinal tubulation. Investig Ophthalmol Vis Sci. 2015;56:2243-53.

10. Dolz-Marco R, Litts KM, Tan ACS, Freund KB, Curcio CA. The evolution of outer retinal tubulation, a neurodegeneration and gliosis prominent in macular diseases. Ophthalmology. 2017;124:1353-67.

11. Perry VH, Cowey A. The lengths of the fibres of Henle in the retina of macaque monkeys: implications for vision. Neuroscience. 1988;25:225-36.

12. Li M, Huisingh C, Messinger J, Dolz-Marco R, Ferrara D, Freund $\mathrm{KB}$, et al. Histology of geographic atrophy secondary to agerelated macular degeneration: a multilayer approach. Retina. 2018;38:1937-53.

13. Bringmann A, Syrbe S, Görner K, Kacza J, Francke M, Wiedemann P, et al. The primate fovea: structure, function and development. Prog Retin Eye Res. 2018;66:49-84.
14. Dolz-Marco R, Balaratnasingam C, Messinger JD, Li M, Ferrara $\mathrm{D}$, Freund $\mathrm{KB}$, et al. The border of macular atrophy in age-related macular degeneration: a clinicopathologic correlation. Am J Ophthalmol. 2018;193:166-77.

15. Preti RC, Govetto A, Filho RGA, Cabral Zacharias L, Gianotti Pimentel S, Takahashi WY, et al. Optical coherence tomography analysis of outer retinal tubulations: Sequential evolution and pathophysiological insights. Retina. 2018;38:1518-25.

16. Sarks SH. Ageing and degeneration in the macular region: a clinico-pathological study. Br J Ophthalmol. 1976;60:324-41.

17. Fleckenstein M, Charbel Issa P, Helb HM, Schmitz-Valckenberg S, Finger RP, Scholl HP, et al. High-resolution spectral domainOCT imaging in geographic atrophy associated with age-related macular degeneration. Investig Ophthalmol Vis Sci. 2008;49:4137-44.

18. Omri S, Omri B, Savoldelli M, Jonet L, Thillaye-Goldenberg B, Thuret G, et al. The outer limiting membrane (OLM) revisited: clinical implications. Clin Ophthalmol. 2010;4:183-95.

19. Guymer RH, Rosenfeld PJ, Curcio CA, Holz FG, Staurenghi G, Freund $\mathrm{KB}$, et al. Incomplete retinal pigment epithelial and outer retinal atrophy in age-related macular degeneration: classification of atrophy meeting report 4. Ophthalmology. 2020;127:394-409.

20. Guidry C, Medeiros NE, Curcio CA. Phenotypic variation of retinal pigment epithelium in age-related macular degeneration. Investig Ophthalmol Vis Sci 2002;43:267-73.

21. Wu KH, Madigan MC, Billson FA, Penfold PL. Differential expression of GFAP in early v late AMD: a quantitative analysis. Br J Ophthalmol. 2003;87:1159-66.

22. Oishi A, Hata M, Shimozono M, Mandai M, Nishida A, Kurimoto Y. The significance of external limiting membrane status for visual acuity in age-related macular degeneration. Am J Ophthalmol. 2010;150:27-32.e1.

23. Chhablani J, Kim JS, Freeman WR, Kozak I, Wang HY, Cheng L. Predictors of visual outcome in eyes with choroidal neovascularization secondary to age related macular degeneration treated with intravitreal bevacizumab monotherapy. Int $\mathrm{J}$ Ophthalmol. 2013;6:62-6.

24. Shin HJ, Chung H, Kim HC. Association between foveal microstructure and visual outcome in age-related macular degeneration. Retina. 2011;31:1627-36.

25. Roberts P, Mittermueller TJ, Montuoro A, Sulzbacher F, Munk M, Sacu S, et al. A quantitative approach to identify morphological features relevant for visual function in ranibizumab therapy of neovascular AMD. Investig Ophthalmol Vis Sci. 2014;55:6623-30.

26. Li M, Dolz-Marco R, Messinger JD, Sloan KR, Ferrara D, Curcio $\mathrm{CA}$, et al. Clinicopathologic correlation of aneurysmal type 1 neovascularization in age-related macular degeneration. Ophthalmol Retina. 2019;3:99-111.

27. Pang CE, Messinger JD, Zanzottera EC, Freund KB, Curcio CA. The onion sign in neovascular age-related macular degeneration represents cholesterol crystals. Ophthalmology. 2015;122:2316-26.

28. Coxeter HSM. Introduction to geometry, 2nd edn. New York: Wiley, 1969.

29. Zanzottera EC, Ach T, Huisingh C, Messinger JD, Freund KB, Curcio CA. Visualizing retinal pigment epithelium phenotypes in the transition to atrophy in neovascular age-related macular degeneration. Retina. 2016:S26-39.

30. Edwards MM, McLeod DS, Bhutto IA, Grebe R, Duffy M, Lutty GA. Subretinal glial membranes in eyes with geographic atrophy. Investig Ophthalmol Vis Sci. 2017;58:1352-67.

31. Green WR, Key SN. Senile macular degeneration: a histopathologic study. Trans Am Ophthalmol Soc. 1977;75:180-254.

32. Grossniklaus HE, Wilson DJ, Bressler SB, Bressler NM, Toth CA, Green WR, et al. Clinicopathologic studies of eyes that were obtained postmortem from four patients who were enrolled in the 
submacular surgery trials: SST Report No. 16. Am J Ophthalmol. 2006;141:93-104.

33. Sarks J, Tang K, Killingsworth M, Arnold J, Sarks S. Development of atrophy of the retinal pigment epithelium around disciform scars. Br J Ophthalmol. 2006;90:442-6.

34. Klein ML, Wilson DJ. Clinicopathologic correlation of choroidal and retinal neovascular lesions in age-related macular degeneration. Am J Ophthalmol. 2011;151:161-9.

35. Grossniklaus HE, Gass JD. Clinicopathologic correlations of surgically excised type 1 and type 2 submacular choroidal neovascular membranes. Am J Ophthalmol. 1998;126:59-69.

36. Lafaut BA, Aisenbrey S, Van den Broecke C, Bartz-Schmidt KU, Heimann K. Polypoidal choroidal vasculopathy pattern in agerelated macular degeneration: a clinicopathologic correlation. Retina. 2000;20:650-4.

37. Grossniklaus HE, Miskala PH, Green WR, Bressler SB, Hawkins BS, Toth C, et al. Histopathologic and ultrastructural features of surgically excised subfoveal choroidal neovascular lesions: submacular surgery trials report No. 7. Arch Ophthalmol. 2005;123:914-21.

38. Curcio CA, Medeiros NE, Millican CL. Photoreceptor loss in agerelated macular degeneration. Investig Ophthalmol Vis Sci. 1996;37:1236-49.

39. Lewis H, Resnick SC, Flannery JG, Straatsma BR. Tissue plasminogen activator treatment of experimental subretinal hemorrhage. Am J Ophthalmol. 1991;111:197-204.

40. Hochman MA, Seery CM, Zarbin MA. Pathophysiology and management of subretinal hemorrhage. Surv Ophthalmol. 1997;42:195-213.

41. Kim JH, Chang YS, Kim JW, Lee TG, Kim CG, Lee DW. Radiating hemorrhage in exudative age-related macular degeneration. Jpn J Ophthalmol. 2016;60:466-75.

42. Au A, Hou K, Baumal CR, Sarraf D. Radial hemorrhage in Henle layer in macular telangiectasia type 2. JAMA Ophthalmol. 2018;136:1182-5.

43. Sato T, Suzuki M, Ooto S, Spaide RF. Multimodal imaging findings and multimodal vision testing in neovascular age-related macular degeneration. Retina. 2015;35:1292-302.

44. Ueda-Arakawa N, Tsujikawa A, Yamashiro K, Ooto S, Tamura H, Yoshimura N. Visual prognosis of eyes with submacular hemorrhage associated with exudative age-related macular degeneration. Jpn J Ophthalmol. 2012;56:589-98.

45. Ito S, Miyamoto N, Ishida K, Kurimoto Y. Association between external limiting membrane status and visual acuity in diabetic macular oedema. Br J Ophthalmol. 2013;97:228-32.

46. Burke TR, Yzer S, Zernant J, Smith RT, Tsang SH, Allikmets R. Abnormality in the external limiting membrane in early Stargardt disease. Ophthalmic Genet. 2013;34:75-7.
47. Keane PA, Patel PJ, Ouyang Y, Chen FK, Ikeji F, Walsh AC, et al. Effects of retinal morphology on contrast sensitivity and reading ability in neovascular age-related macular degeneration. Investig Ophthalmol Vis Sci. 2010;51:5431-7.

48. Schmidt-Erfurth U, Waldstein SM. A paradigm shift in imaging biomarkers in neovascular age-related macular degeneration. Prog Retin Eye Res. 2016;50:1-24.

49. Kashani AH, Lebkowski JS, Rahhal FM, Avery RL, Salehi-Had $\mathrm{H}$, Dang W, et al. A bioengineered retinal pigment epithelial monolayer for advanced, dry age-related macular degeneration. Sci Transl Med. 2018;10:eaao4097.

50. Moriyama M, Ohno-Matsui K, Shimada N, Hayashi K, Kojima A, Yoshida T, et al. Correlation between visual prognosis and fundus autofluorescence and optical coherence tomographic findings in highly myopic eyes with submacular hemorrhage and without choroidal neovascularization. Retina. 2011;31:74-80.

51. Li M, Dolz-Marco R, Huisingh C, Messinger JD, Feist RM, Ferrara D, et al. Clinicopathologic correlation of geographic atrophy secondary to age-related macular degeneration. Retina. 2019;39:802-16.

52. Asai T, Ikuno Y, Nishida K. Macular microstructures and prognostic factors in myopic subretinal hemorrhages. Investig Ophthalmol Vis Sci. 2014;55:226-32.

53. Lee K, Park YG, Park YH. Visual prognosis after pneumatic displacement of submacular hemorrhage according to age-related macular degeneration subtypes. Retina. 2020 [Epub ahead of print].

54. Schmidt-Erfurth U, Laqua H, Schlötzer-Schrehard U, Viestenz A, Naumann GO. Histopathological changes following photodynamic therapy in human eyes. Arch Ophthalmol. 2002;120:835-44.

55. Reinke MH, Canakis C, Husain D, Michaud N, Flotte TJ, Gragoudas ES, et al. Verteporfin photodynamic therapy retreatment of normal retina and choroid in the cynomolgus monkey. Ophthalmology. 1999; 106:1915-23.

56. Husain D, Kramer M, Kenny AG, Michaud N, Flotte TJ, Gragoudas ES, et al. Effects of photodynamic therapy using verteporfin on experimental choroidal neovascularization and normal retina and choroid up to 7 weeks after treatment. Investig Ophthalmol Vis Sci. 1999;40:2322-31.

57. Chen L, Messinger JD, Sloan KR, Swain TA, Sugiura Y, Yannuzzi LA, et al. Non-exudative neovascularization supporting outer retina in age-related macular degeneration, a clinicopathologic correlation. Ophthalmology. 2020. [Epub ahead of print]

58. Chen L, Li M, Messinger JD, Ferrara D, Curcio CA, Freund KB. Recognizing atrophy and mixed-type neovascularization in agerelated macular degeneration via clinicopathologic correlation. Transl Vis Sci Technol. 2020. in press. 